\title{
Characterizing shock waves in hydrogel using high speed imaging and a fiber-optic probe hydrophone
}

Phillip A. Anderson, M. R. Betney, H. W. Doyle, B. Tully, Y. Ventikos, N. A. Hawker, and Ronald A. Roy

Citation: Physics of Fluids 29, 057101 (2017); doi: 10.1063/1.4982062

View online: http://dx.doi.org/10.1063/1.4982062

View Table of Contents: http://aip.scitation.org/toc/phf/29/5

Published by the American Institute of Physics

\section{Articles you may be interested in}

Many-body dissipative particle dynamics modeling of fluid flow in fine-grained nanoporous shales

Physics of Fluids 29, 056601 (2017); 10.1063/1.4981136

Streamwise vortex breakdown in supersonic flows

Physics of Fluids 29, 054102 (2017); 10.1063/1.4982901

Finite response time of shock wave modulation by turbulence

Physics of Fluids 29, 051701 (2017); 10.1063/1.4982932

Breakup of a liquid rivulet falling over an inclined plate: Identification of a critical Weber number

Physics of Fluids 29, 052101 (2017); 10.1063/1.4981920

Interactions of a co-rotating vortex pair at multiple offsets

Physics of Fluids 29, 057102 (2017); 10.1063/1.4982217

Effects of heat sink and source and entropy generation on MHD mixed convection of a Cu-water nanofluid in a lid-driven square porous enclosure with partial slip

Physics of Fluids 29, 052001 (2017); 10.1063/1.4981911

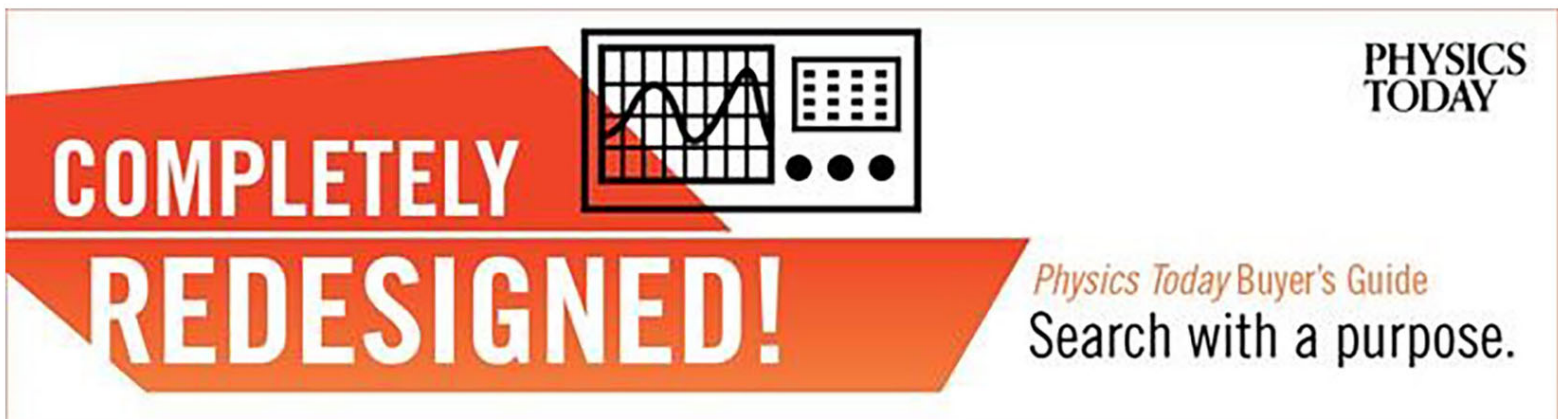




\title{
Characterizing shock waves in hydrogel using high speed imaging and a fiber-optic probe hydrophone
}

\author{
Phillip A. Anderson, ${ }^{1}$ M. R. Betney, ${ }^{1, a)}$ H. W. Doyle, ${ }^{2}$ B. Tully, ${ }^{2}$ Y. Ventikos ${ }^{3}$ N. A. Hawker, ${ }^{2}$ \\ and Ronald A. Roy ${ }^{1}$ \\ ${ }^{1}$ Department of Engineering Science, University of Oxford, Oxford OX1 3PJ, United Kingdom \\ ${ }^{2}$ First Light Fusion, Ltd., 10 Oxford Industrial Park, Mead Rd., Yarnton, Kidlington, \\ Oxfordshire OX5 1QU, United Kingdom \\ ${ }^{3}$ Department of Mechanical Engineering, University College London, London WC1E 7JE, United Kingdom
}

(Received 14 February 2017; accepted 11 April 2017; published online 1 May 2017)

\begin{abstract}
The impact of a stainless steel disk-shaped projectile launched by a single-stage light gas gun is used to generate planar shock waves with amplitudes on the order of $10^{2} \mathrm{MPa}$ in a hydrogel target material. These shock waves are characterized using ultra-high-speed imaging as well as a fiber-optic probe hydrophone. Although the hydrogel equation of state (EOS) is unknown, the combination of these measurements with conservation of mass and momentum allows us to calculate pressure. It is also shown that although the hydrogel behaves similarly to water, the use of a water EOS underpredicts pressure amplitudes in the hydrogel by $\sim 10 \%$ at the shock front. Further, the water EOS predicts pressures approximately $2 \%$ higher than those determined by conservation laws for a given value of the shock velocity. Shot to shot repeatability is controlled to within $10 \%$, with the shock speed and pressure increasing as a function of the velocity of the projectile at impact. Thus the projectile velocity may be used as an adequate predictor of shock conditions in future work with a restricted suite of diagnostics. Published by AIP Publishing. [http://dx.doi.org/10.1063/1.4982062]
\end{abstract}

\section{INTRODUCTION}

Shock waves are ubiquitous in nature and play an important role in a variety of scientific processes and industrial applications such as equation of state (EOS) measurements, ${ }^{1-3}$ medical treatments,, 45 explosives, ${ }^{6,7}$ droplet impact studies, ${ }^{8-10}$ cavitation studies, ${ }^{6,7,11}$ and material characterization. Techniques for reproducibly generating and characterizing shocks enable the improved understanding of relevant physics and facilitate the application of shock phenomena to problems in science and technology.

In our case, we are interested in developing a platform for studying shock-driven cavity collapse that is similar to previous work in this area. ${ }^{6,7,11}$ We describe a system for the generation of shock waves within a hydrogel target, and efforts to characterize the shock for use in future studies. Hydrogel has properties similar to water but offers advantages as a target material. Because hydrogels hold their shape, inclusions such as cylindrical or spherical cavities may be cast within them and retained. In contrast, inclusions in water and other liquids are difficult to maintain due to buoyancy and dissolution, and surface tension also restricts readily achievable shapes to spheres. Unfortunately, the detailed rheology and equation of state of most hydrogels are not well known, and this can impede efforts to match experimental results with a hydrodynamic model. We address this issue in Section III D.

The most commonly used methods to generate shock waves in a medium are explosives, laser ablation, pulsed-power electromagnetic actuation, and high-velocity

a)Electronic mail: matthew.betney@ firstlightfusion.com projectile impacts. The range of shock conditions that can be achieved from each of these methods is different, and not all are applicable in the context of shock-driven cavity collapse. The amplitude and duration of the elevated pressure condition trailing the shock front are important parameters to measure and control. Sustained high pressure on the order of, or greater than, the collapse time of the cavity is desired.

Shocks generated by the impact of a projectile launched by a light gas gun (LGG) offer many advantages for this application and have been used in the past. With a single-stage LGG, velocities on the order of $1 \mathrm{~km} \mathrm{~s}^{-1}$ are achievable. At such velocities, peak pressures of order $10^{2} \mathrm{MPa}$ to $10^{3} \mathrm{MPa}$ can be generated, depending on the material properties of the impactor and target. Two-stage LGGs are capable of launching projectiles ten times faster than their single-stage counterparts, with a corresponding increase in pressure amplitude. Laser-generated shocks can reach much higher pressures, but they have a profile similar to a blast-wave which decays very quickly. With a projectile impact, the duration of the high pressure after the shock front is much longer, on the order of $2 l c$, where $l$ is the length of the projectile and $c$ is the sound speed in the projectile material. LGGs avoid issues of preheating ${ }^{12}$ and are suitable for generating broad planar shock fronts.

Five variables are relevant to describing the state behind a shock front: pressure $(P)$, density $(\rho)$, internal energy $(E)$, shock velocity $\left(U_{s}\right)$, and particle velocity $\left(u_{p}\right)$. With conservation of mass, momentum, and energy, only two of the five variables are required to define the behind state, ${ }^{12}$ and if an appropriate equation of state (EOS) is known for the 
material one variable is sufficient. Typically, shock velocity and particle velocity are the chosen observables, ${ }^{12,13}$ although density is sometimes measured instead. Many robust techniques already exist to measure shock velocity either directly or indirectly, such as time-resolved Schlieren imaging, ${ }^{14}$ velocity interferometry (VISAR), ${ }^{15,16}$ and fiber-optic shock break out (FOSBO). ${ }^{2}$ Particle velocity is often calculated from $U_{s}$ and the velocity of the projectile by shock-impedance matching. ${ }^{12,17,18}$ Density can be inferred non-invasively using wave reverberation ${ }^{3,13}$ or by inserting a probe such as the fiber-optic probe hydrophone (FOPH). ${ }^{19-21}$

In this work, a single-stage LGG is employed to generate shock waves in a solid block of hydrogel. We measure $U_{s}$ and planarity of the shock front in the hydrogel using high-speed Schlieren photography. Temporally resolved point measurements of the index of refraction $(n)$ in the hydrogel are made with a purpose-built FOPH and converted to density through the Gladstone-Dale relationship. ${ }^{19,22-24}$ Details of the experiment are given in Section II, followed by illustrative results (Sec. III A), characterization of shock repeatability (Sec. III B), characterization of the shock profile at varying positions (Sec. III C), and the effect of varying projectile velocity (Sec. III D).

\section{EXPERIMENTAL METHODOLOGY}

A schematic of the experimental setup is shown in Figure 1. A single-stage LGG accelerates a projectile towards the target assembly, typically with nitrogen or helium as the working gas. The gun barrel is $5.2 \mathrm{~m}$ long with a $70 \mathrm{~mm}$ bore diameter. Dual bursting diaphragms in the breech allow the

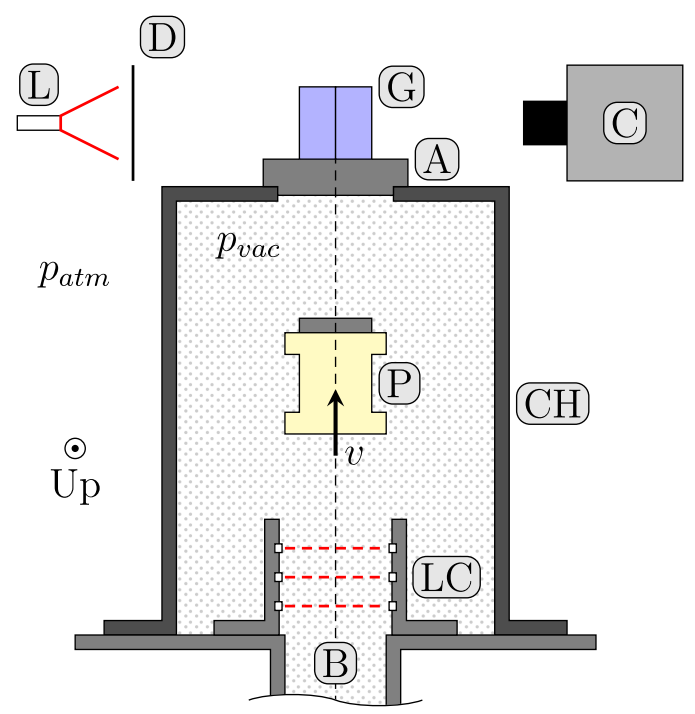

FIG. 1. Top-down schematic of the target area. An enlarged chamber $(\mathrm{CH})$ encloses the end of the gun barrel (B) and is sealed on the far side by the aluminum disc (A). The seal allows the chamber and barrel to be evacuated to $p_{v a c}$, while the outer region remains at atmospheric pressure. The projectile (P) crosses three light curtains (LC) upon exiting the barrel, which allows the velocity just before impact $(v)$ to be determined. At impact, a shock is generated in the aluminum disc and propagates through to the hydrogel $(\mathrm{G})$. The high speed camera (C) is positioned to image through the side of the hydrogel and is back lit with an illumination laser (L) and diffuser (D). The FOPH (not shown) is inserted into the hydrogel from the top surface. reservoir pressure to be varied as desired. With the barrel evacuated to $0.4 \mathrm{kPa}$, a $0.5 \mathrm{~kg}$ projectile can be accelerated up to $700 \mathrm{~m} \mathrm{~s}^{-1}$. Evacuating the barrel not only yields higher projectile velocities, but also prevents the target assembly from being disturbed by an air blast prior to impact, and eliminates light flashes from gas compressed between the projectile and aluminum disc. Three light curtains positioned at the end of the barrel provide timings needed to calculate projectile velocity.

The projectile is composed of a $15 \mathrm{~mm}$ thick, $50 \mathrm{~mm}$ diameter steel disc embedded in a nylon sabot. The sabot diameter matches the bore of the barrel and is waisted in the middle to reduce overall mass without weakening to the point of fracturing in the barrel. No sabot stripper is used. After the projectile exits the barrel, it travels $450 \mathrm{~mm}$ in free flight through an evacuated chamber before impacting the target assembly.

The target assembly consists of an aluminum disc, hydrogel cube, and plastic support tray. The aluminum disc is $24 \mathrm{~mm}$ thick and of diameter $102 \mathrm{~mm}$. The impact face of the disc seals against the chamber with an o-ring so that the barrel may be evacuated and provides a guide for alignment against locating pins on the chamber. Additionally, the aluminum disc serves as an impedance matching layer between the projectile and the hydrogel, as discussed at the end of this section. Two threaded rods (not shown) extend from the rear face of the disc and hold a support tray (not shown). The hydrogel rests on the tray and is slid into contact with the aluminum. Care is taken to ensure that no air bubbles are trapped between the hydrogel and aluminum.

The hydrogel is made by mixing a ratio of $0.5 \mathrm{~g}$ of gellan gum (Phytagel, Sigma Aldrich P8169-100G) and $0.05 \mathrm{~g}$ of $\mathrm{CaCl}$ per $100 \mathrm{~g}$ of water. The mixture is heated to near boiling until combined and then poured into molds where it sets upon cooling. While we only discuss solid hydrogel blocks in the current paper, occlusions such as cylindrical and spherical cavities can be created in the hydrogel by stacking multiple slabs. Hydrogel dimensions are $50 \times 50 \times 42 \mathrm{~mm}^{3}$, with the short dimension co-axial to the gun barrel. These dimensions ensure a substantial 1-D region of shock propagation in the hydrogel, and it is within this region that we are most interested in taking measurements.

Shock propagation through the hydrogel is imaged with a ultra-high-speed camera (Specialised Imaging SimX-16, 200 Mfps maximum, 16 frames at $1280 \times 960$ pixels, 12 bit dynamic range, Sigma 70-300 mm F4-5.6 DG Macro lens with $1 \mathrm{in}$. extension) to determine $U_{s}$ and planarity of the shock front. Images are back lit with a diode laser (Cavitar Cavilux, $200 \mathrm{~W}, 650 \mathrm{~nm}$ center wavelength, $7 \mathrm{~nm}$ FWHM, $24 \mu$ s pulse width), and a $100 \mathrm{~mm}, 120$ grit plate diffuser (Edmund Optics). The imaging axis is perpendicular to the direction of shock propagation, so that the shock is seen traveling left to right across the image. Because the SimX camera uses sixteen physically separate CCDs, digital post-processing is necessary to align the images. Postprocessing is done with the Insight ToolKit (ITK) and transformations determined from a static image sequence. Spatial calibration is determined from a resolution target (Thorlabs R2L2S1P). 
The restriction to sixteen frames per event necessitates a reliable, low-jitter trigger. Although diagnostics with long exposures or very large recording times may be triggered from the light curtain signal as the projectile passes the end of the barrel, this method of triggering is generally unsuitable in the current experiment. Given the relatively long free flight distance between the light curtains and the target, mis-predicting the projectile velocity by as little as $1 \%$ would result in an uncertainty for the impact time on the order of $10^{-5} \mathrm{~s}$. By comparison, this is equivalent to the time required for the shock to travel halfway across the hydrogel. Thus the light curtains are unsuitable, and instead a method has been devised to trigger from the moment of impact. Two strips of copper shim $(\sim 70 \mathrm{~mm} \times 4 \mathrm{~mm} \times 0.2 \mathrm{~mm})$ are adhered to, but insulated from, the impact face of the aluminum disc with double sided tape. The copper strips are parallel to each other and positioned so that they span the center of the impact area but also extend beyond the aluminum disc. The trailing ends are connected to a monostable trigger circuit designed to emit a single TTL pulse when the electrical impedance between the strips drops from high to low. Upon impact, the steel projectile bridges the copper strips and closes the circuit. With this method, jitter in the trigger supplied to the diagnostics has been reduced to $<300 \mathrm{~ns}$.

A FOPH provides a means to record the local refractive index in a material with high temporal resolution. The sensing area is the end of a clean, cleaved optical fiber $(\varnothing 100 \mu \mathrm{m})$ inserted directly into the hydrogel from the top surface and angled towards the aluminum plate. We employ a custombuilt FOPH based on the design reported by Parsons and Fowlkes, ${ }^{20}$ and suitable performance was verified using calibrated oils as described by Arvengas. ${ }^{21}$ The FOPH laser is a $1000 \mathrm{~mW}$ fiber-pigtailed diode laser with a center wavelength of $860 \mathrm{~nm}$ and $3.1 \mathrm{~nm}$ spectral width (QPhotonics QLFD-850-1000M), and the detector is a $150 \mathrm{MHz}$ broadband avalanche photo-detector (Thorlabs PDA10A) with a rise time of $2.3 \mathrm{~ns}$. A $300 \mathrm{MHz}, 2.5 \mathrm{GSample} \mathrm{s}^{-1}$ oscilloscope (Tektronix DPO3034) is used to digitize data from the FOPH.

At the time of impact, both the ultra-high-speed camera and the FOPH oscilloscope are directly triggered using the method described above. Timings for the images and illumination are set by independently programmable delays within the ultra-high-speed camera, and the oscilloscope has sufficient recording time to capture the entire event, so requires no additional delay. Since both diagnostics are referenced to a common $t_{0}$ (corresponding to the time of projectile impact), they are synchronized with each other.

Refractive index, as measured by the FOPH, can be converted to density using the Gladstone-Dale relation, although this is limited to pressures less than $500 \mathrm{MPa} .{ }^{20}$ Typically, density is then converted to pressure using an equation of state for the material. For water, the equation of state commonly used is the Tait equation,

$$
p_{1}=\left(Q+p_{o}\right)\left(\frac{\rho_{1}}{\rho_{o}}\right)^{\gamma}-Q,
$$

where $\gamma=7.44, p_{o}=0.1 \mathrm{MPa}$, and $Q=295.5 \mathrm{MPa} .{ }^{20}$ In this work, we use the Tait equation as a first approximation but, because shock velocity is known from the high-speed images, density can be converted to pressure by using conservation of mass and momentum,

$$
p_{1}=p_{o}+\frac{\rho_{o}}{\rho_{1}}\left(\rho_{1}-\rho_{o}\right) U_{s}^{2},
$$

keeping in mind that this conversion is only strictly true at the shock front, and merely an approximation in the shocked region.

We conclude by returning to the discussion of the aluminum disc as an impedance matching layer. In the work of Dear, Bourne, and Field, ${ }^{6,9}$ a flyer plate projectile directly impacts the gelatin target, and shock amplitude was calculated from the projectile velocity and specific acoustic impedances using

$$
P=v \cdot \frac{Z_{1} Z_{2}}{Z_{1}+Z_{2}}
$$

where $P$ is the peak pressure, $v$ is the projectile velocity, and the specific acoustic impedance is the product of material density and sound speed, $Z_{i}=\rho_{i} c_{i}$. The large impedance mismatch between the flyer material and gelatin results in a relatively inefficient conversion to pressure, which is improved by adding an impedance matching layer between the projectile and hydrogel. Pressure in the impedance matching layer is governed by Equation (3) and is scaled down by a transmission factor $(T)$ going into the hydrogel. The transmission factor is given by the standard relationship for the transmission and reflection of a plane acoustic wave normally incident on a plane interface, ${ }^{25}$ Equation (4),

$$
T=\frac{2 Z_{2}}{Z_{1}+Z_{2}} .
$$

Note that in Equations (3) and (4), subscripts 1 and 2 refer to the material before and after the relevant interface, respectively. Under these assumptions, an aluminum matching layer between a steel projectile and hydrogel target $(Z$ is assumed to be that of water) results in a roughly $30 \%$ gain in achieved pressure. Although the shock pressure is not measured at the aluminum-hydrogel interface, we present evidence of an increase in peak pressure by incorporating the impedance matching layer.

\section{RESULTS}

\section{A. Illustrative results and basic analysis}

Figure 2 shows the results from a typical high-speed image sequence, including a single full frame of the sequence (Figure 2(a)), and a composite built from stacked slices of each frame (Figure 2(b)). The projectile velocity for this shot was 451 $\pm 6 \mathrm{~m} \mathrm{~s}^{-1}$. The shock front appears dark in the back-lit images and travels from left to right through the hydrogel before reaching the FOPH tip, which is circled in the figure. Velocity of the shock front is directly measured from the image sequence. Behind the shock front are alternating light and dark bands, which result from surface waves, that obscure the interior of the hydrogel.

In addition to shock velocity, the curvature of the shock front is readily obtainable from the sequence of high-speed images. Figure 3 shows the measured curvature of the shock front from the same sequence used to construct Figure 2(a), 


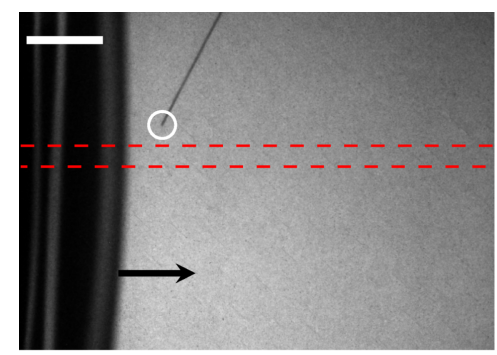

(a)

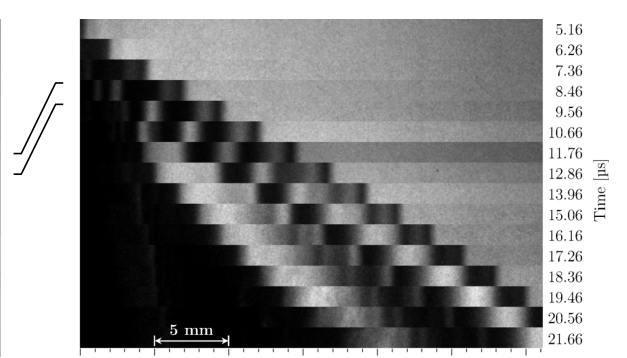

(b)

FIG. 2. A representative result from the SimX-16 high-speed camera. From this view, the projectile impacts to the left of the frame, and the shock propagates from left to right in the solid hydrogel block. Projectile velocity before impact was $451 \pm 6 \mathrm{~m} \mathrm{~s}^{-1}$. (a) A single frame from the high-speed sequence. The shock wave has already entered the hydrogel and travels from left to right, as indicated by the arrow. The sensing tip of the FOPH is circled, and the white bar indicates $5 \mathrm{~mm}$. (b) Stacked horizontal slices from the high-speed sequence showing the shock wave propagating across the hydrogel. The fourth slice was taken from (a) as indicated by the dashed lines.

where the curvature $\kappa$ is the reciprocal of the radius of a circle fit to the shock front. The front is initially near planar $(\kappa \approx 0)$ and quickly develops a more pronounced curvature leading to a more or less constant value $(\kappa \approx 11)$.

A representative measurement from the FOPH (from the same shot as shown above for the $\operatorname{SimX}$ ) is shown in Figures 4 and 5. For this measurement, the sensing tip of the FOPH was centered on the barrel axis in the hydrogel and $10 \mathrm{~mm}$ away from the surface of the aluminum disc. As the figures show, the shock arrives at the FOPH $9.5 \mu$ s after the impact. This propagation time matches expectations based on the thickness and shock velocity of aluminum and hydrogel. The measured rise time of the shock front is $30 \mathrm{~ns}$, which corresponds to the shock transit time across the fiber tip, followed by a sustained high pressure lasting tens of microseconds. The secondary rise in pressure seen $\sim 4 \mu$ s after the arrival of the shock front is a persistent feature in these measurements and is consistent with reflections within the steel projectile. Typically, the high-pressure tail is interrupted at later times by the onset of large fluctuations in the signal (not shown) before the signal recovers to ambient levels. These fluctuations are presumed to be caused by damage to the fiber itself as the shock travels past.

Figure 5 depicts the data after conversion to pressure using both the Tait EOS (Equation (1)) and conservation of mass and momentum (Equation (2)) for comparison. Although conversion to pressure using the conservation laws only applies

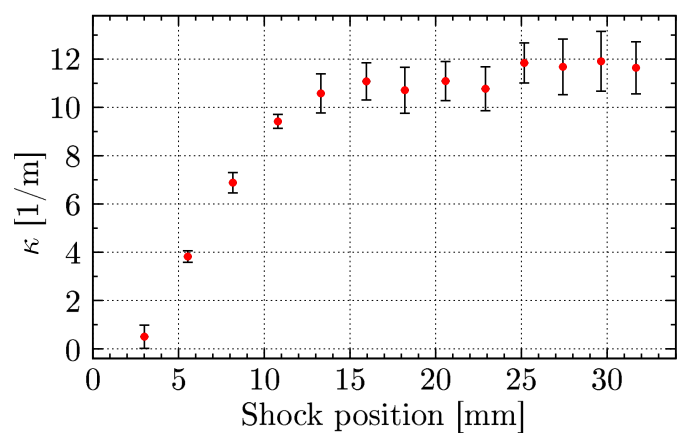

FIG. 3. Curvature of the shock front as it propagates across the hydrogel, as measured from the SimX images. Error bars indicate one standard deviation in the measurement. The shock front quickly evolves from near planar $(\kappa \approx 0)$ at the aluminum interface to a constant curvature, $\kappa \approx 11$. at the shock front, as noted above, the shock profiles determined by the two methods are consistently similar. We find that the Tait EOS consistently underpredicts the peak pressure by $\sim 10 \%$ as compared to conversion using the conservation relations. This highlights the material differences between water and the hydrogel and suggests that, while the Tait EOS is a reasonable first approximation, it is not a suitable conversion in this case. For this reason, we rely on Equation (2) to convert from density to pressure for all pressure values reported in this paper unless explicitly stated otherwise.

As a check on the FOPH results, independent measurements of the baseline density in hydrogel were undertaken. Baseline density was found to be $1010 \pm 7 \mathrm{~kg} \mathrm{~m}^{-3}$ by these measurements, which agrees well with $1012 \pm 13 \mathrm{~kg} \mathrm{~m}^{-3}$ as determined from FOPH measurements. Small-signal sound speed of the hydrogel was also measured directly using a pulseecho technique and was found to agree with values for pure water under the same conditions.

\section{B. Shock repeatability}

Before any meaningful comparisons can be made between shots, repeatability of the system needs to be established. To that end, we compare four shots taken under the same experimental conditions. For each shot, the FOPH tip was positioned

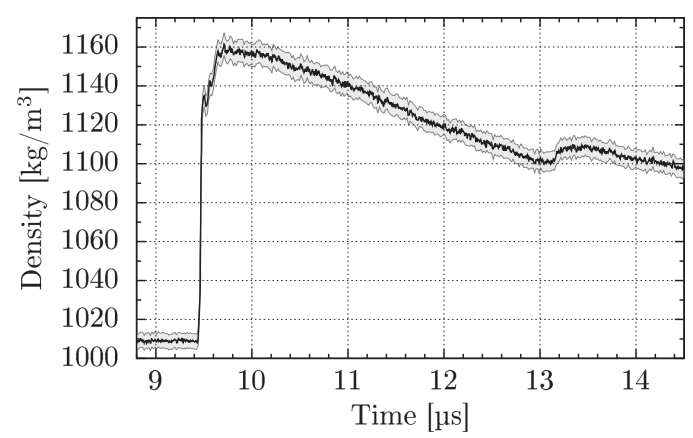

FIG. 4. Representative FOPH measurement converted to density using the Gladstone-Dale relation, with $68 \%$ confidence bounds indicated by the shaded area. The sensing tip was centered on the barrel axis and placed $10 \mathrm{~mm}$ from the surface of the aluminum disc. Rise time of the shock front is $30 \mathrm{~ns}$, followed by a sustained high density. The secondary bump in density after $t=13 \mu \mathrm{s}$ is a consistent feature in these measurements. 


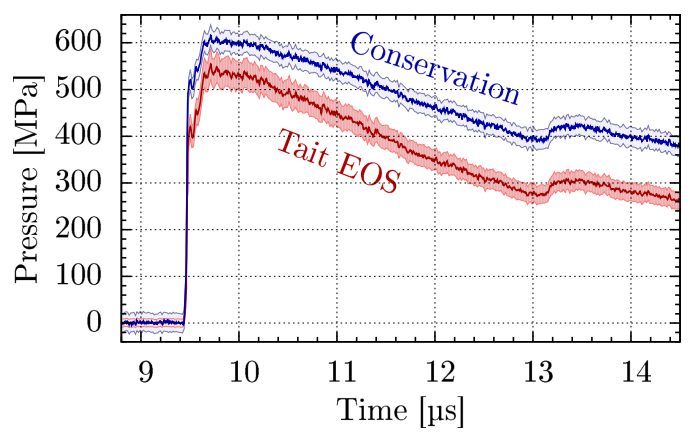

FIG. 5. Representative FOPH measurement converted to pressure using the Tait EOS (red) and conservation of mass and momentum (blue) for the same shot as presented in Figure 4. 68\% confidence bounds are indicated by the shaded areas.

in the hydrogel along the barrel axis and $10 \mathrm{~mm}$ from the aluminum disc. Projectile velocity was $394 \pm 8 \mathrm{~m} \mathrm{~s}^{-1}$. The density jump associated with the arrival of the shock was repeatable to about $10 \%$, with an amplitude of $\langle\Delta \rho\rangle=140 \pm 15 \mathrm{~kg} \mathrm{~m}^{-3}$, and the profile of the shock is very consistent as can be seen in Figure 6. Here, the normalized pressure jump is shown in order to emphasize the shot to shot repeatability of the shock profile.

\section{Shock profile at varying positions}

The shock wave was measured at various hydrophone locations in the hydrogel to determine if and how the temporal profile varied as a function of position. The cartoon in Figure 7 shows locations where the FOPH tip was placed. In particular, we look at the shock profile along the barrel axis at different distances from the aluminum disc and, for a set distance from the disc, the shock profile from off-axis points. For each of these shots, the projectile velocity was fixed at $396 \pm 3 \mathrm{~m} \mathrm{~s}^{-1}$.

Figure 8(a) shows an overlay of FOPH measurements from four different shots taken at different positions along the $x$-axis $(y, z=0)$ and with the propagation delays artificially removed so that the rise of the shocks is aligned. As the shock travels further from the aluminum striker, the main features remain, but with a large drop in peak pressure. We note that the pressure measured after the shock propagates $10 \mathrm{~mm}$ through the hydrogel is the same as is predicted entering the hydrogel for a direct impact by Equation (3). Given the rapid

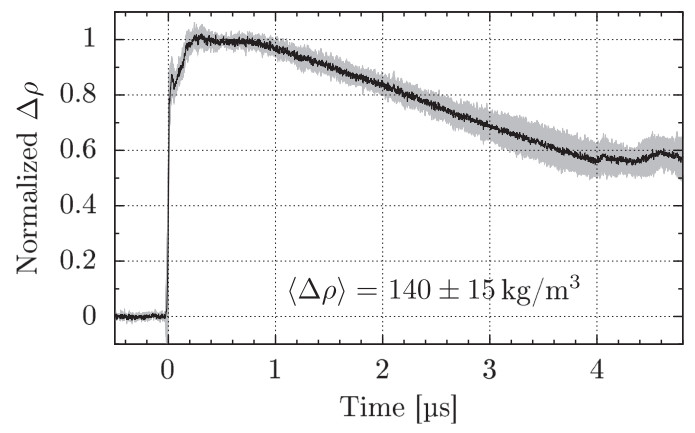

FIG. 6. The mean normalized density jump profile from four comparable shots, with the standard deviation indicated by the shaded area. For each shot, the FOPH tip was on the barrel axis, $10 \mathrm{~mm}$ from the aluminum disc, and projectile velocity was $394 \pm 8 \mathrm{~m} \mathrm{~s}^{-1}$. The average density jump was $\langle\Delta \rho\rangle=140 \pm 15 \mathrm{~kg} \mathrm{~m}^{-3}$.

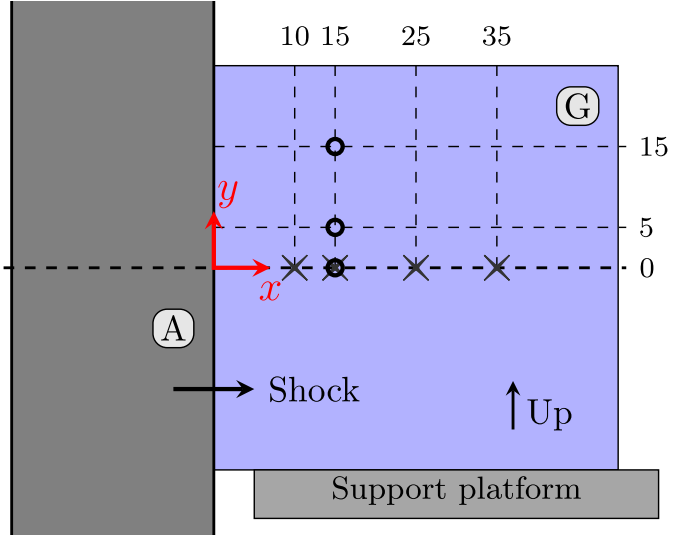

FIG. 7. Side-on cartoon depicting placement of the FOPH sensing tip in the gel. The aluminum disc (A) is on the left, just as in Figure 2. The line $y$, $z=0$ (where $z$ is out of the page) is coaxial with the gun barrel. In all cases, the FOPH tip resides on the $z=0$ plane. Units are $\mathrm{mm}$.

decay in shock amplitude, we can assume that the shock pressure at the aluminum-hydrogel interface is significantly larger, presumably due to the presence of the impedance matching layer.

In contrast to the on-axis results, off-axis measurements $15 \mathrm{~mm}$ from the aluminum disc do not reveal a significant difference in the peak amplitude or overall profile of the shock, as shown in Figure 8(b). Note that, as in Figure 8(a), the slight delays due to shock front curvature have been artificially removed. Both of these figures show examples where the fiber has been damaged at later times, as alluded to in Section III A.

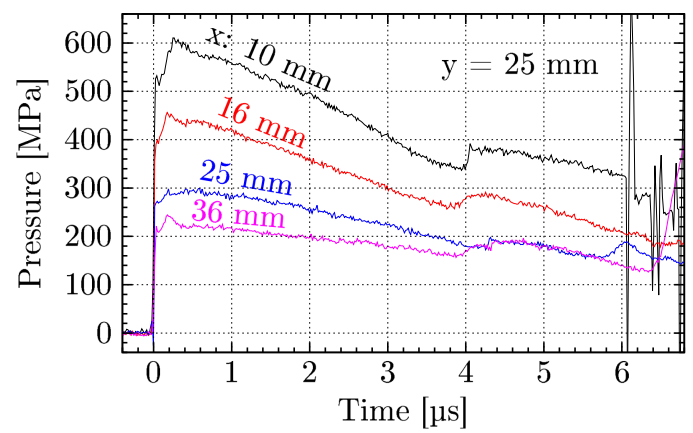

(a)

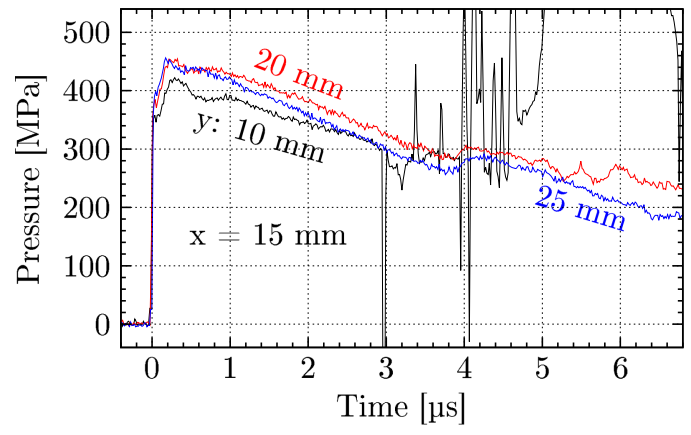

(b)

FIG. 8. Shock profiles at different locations in the gel. Projectile velocity was $396 \pm 3 \mathrm{~m} \mathrm{~s}^{-1}$ for each shot. For clarity, uncertainty bands have been omitted. (a) Comparison of the shock profile at different horizontal positions in the gel, corresponding to $\times$-marks in Figure 7 . (b) Comparison of the shock profile at different vertical ositions in the gel, corresponding to o-marks in Figure 7. 


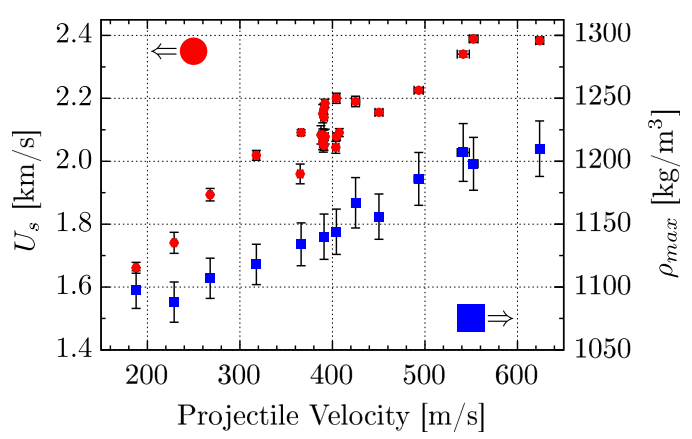

FIG. 9. Shock velocity (red circles) and peak density (blue squares) determined from the high-speed images and $\mathrm{FOPH}$, respectively, against projectile velocity.

\section{Projectile velocity dependence}

In another series of shots, we characterize the shock at a single point of interest in the hydrogel while varying the velocity of the projectile. Again, the FOPH tip was located on the barrel axis, $10 \mathrm{~mm}$ from the aluminum disc. As shown in Section III C, peak pressure diminishes with distance from the aluminum disc due to geometric spreading and dissipation. Thus a compromise must be reached between maximizing pressure and maintaining a reasonable standoff distance from the aluminum disc. The importance of the standoff distance is twofold. First, this ensures that the shock wave has time to steepen after entering the hydrogel. Second, in future work, we intend to cast cavities in the gel, and if the layer of hydrogel separating the cavity from the surface is too thin, there is an increased possibility of damaging the sample or distorting the target geometry during fabrication or deployment. Moreover, such configurations will help validate computational studies of shock-induced cavity collapse. ${ }^{26,27}$

FOPH measurements and high-speed images were captured for varying projectile velocities, and these are summarized in Figure 9. As expected, both $U_{s}$ and $\rho_{\max }$ increase steadily with projectile velocity. Importantly for future studies, the projectile velocity is a reasonable indicator of the shock conditions achieved and may be used to estimate shock conditions for later tests where an invasive measure of density is not practical.

Finally, we can combine the data from Figure 9 to remove the dependency on projectile velocity. The result, shown in Figure 10, shows the peak density against velocity of the shock.

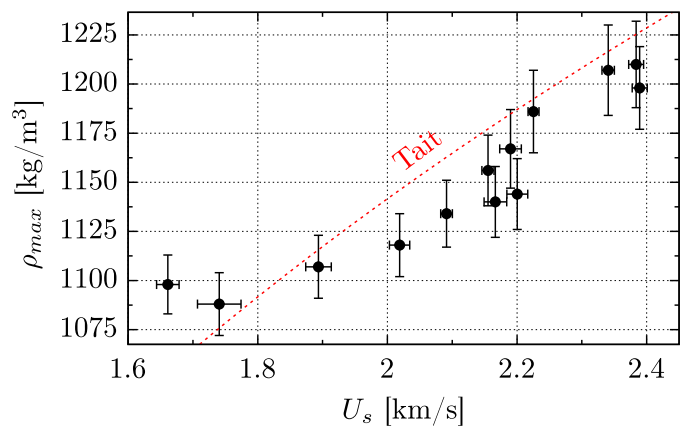

FIG. 10. Individual-shot peak density, measured with the FOPH, plotted versus the corresponding shock velocity derived from the SIMX images.
This type of plot is commonly used in EOS work when characterizing materials. For comparison, the Tait EOS relation is also shown in Fig. 10. This result reinforces our initial assumption that the Tait EOS is suitable as a first approximation for the hydrogel behavior, but also shows that it tends to overpredict the density by about $2 \%$ for a given value of the shock velocity.

\section{CONCLUSIONS}

The light gas gun system is a very flexible system for controlled and repeatable shock wave generation. By using an aluminum matching layer, we are able to generate higher peak pressures and densities in the hydrogel material than a direct impact. Relatively planar shocks are generated across the cross section of the hydrogel. With this particular implementation, peak pressures up to $1 \mathrm{GPa}$ have been measured.

Measurements from the FOPH were converted to pressure using both the Tait EOS and conservation laws. The latter method consistently estimated a larger shock amplitude, but otherwise similar shock profiles.

The measured density jump and pressure amplitude at the shock front can be related to projectile velocity to within $10 \%$ accuracy from projectile velocity. This is important for future studies where direct measurement of density or pressure is not possible. In particular, future studies involving cavities in the hydrogel are not amenable to the FOPH. The presence of a gas body near the FOPH will prevent useful refractive index measurements, and the FOPH fiber is likely to interfere with the cavity collapse dynamics.

These data are also useful for characterizing the hydrogel, which does not have a known EOS. The relationship between $\rho_{\max }$ and $U_{s}$ may benefit hydro simulations in this material.

${ }^{1}$ R. W. Lemke, M. D. Knudson, C. A. Hall, T. A. Haill, P. M. Desjarlais, J. R. Asay, and T. A. Mehlhorn, "Characterization of magnetically accelerated flyer plates," Phys. Plasmas 10(4), 1092-1099 (2003).

${ }^{2}$ M. D. Knudson, R. W. Lemke, D. B. Hayes, C. A. Hall, C. Deeney, and J. R. Asay, "Near-absolute Hugoniot measurements in aluminum to 500 GPa using a magnetically accelerated flyer plate technique," J. Appl. Phys. 94(7), 4420 (2003).

${ }^{3}$ M. D. Knudson, D. L. Hanson, J. Bailey, C. A. Hall, and J. Asay, "Use of a wave reverberation technique to infer the density compression of shocked liquid deuterium to 75 GPa," Phys. Rev. Lett. 90(3), 035505 (2003).

${ }^{4}$ T. G. Leighton and R. O. Cleveland, "Lithotripsy," Proc. Inst. Mech. Eng., Part H 224, 317-342 (2010).

${ }^{5}$ C.-J. Wang, "An overview of shock wave therapy in musculoskeletal disorders," Chang Gung Med. J. 26(4), 220-232 (2003).

${ }^{6}$ J. P. Dear and J. E. Field, "A study of the collapse of arrays of cavities," J. Fluid Mech. 190, 409-425 (1988).

${ }^{7}$ N. K. Bourne, "On the collapse of cavities," Shock Waves 11(6), 447-455 (2002).

8 J. E. Field, M. B. Lesser, and J. P. Dear, "Studies of two-dimensional liquidwedge impact and their relevance to liquid-drop impact problems," Proc. R. Soc. A 401(1821), 225-249 (1985).

${ }^{9}$ J. P. Dear and J. E. Field, "High-speed photography of surface geometry effects in liquid/solid impact," J. Appl. Phys. 63(4), 1015 (1988).

${ }^{10}$ J. E. Field, J. P. Dear, and J. E. Ogren, "The effects of target compliance on liquid drop impact,” J. Appl. Phys. 65(2), 533 (1989).

${ }^{11}$ J. P. Dear, J. E. Field, and A. J. Walton, "Gas compression and jet formation in cavities collapsed by a shock wave," Nature 332, 505-508 (1988).

${ }^{12}$ M. Yokoo, N. Kawai, Y. Hironaka, K. G. Nakamura, and K. I. Kondo, "Diagnostic system to measure spatial and temporal profiles of shock front using compact two-stage light-gas gun and line reflection method," Rev. Sci. Instrum. 78(4), 043904 (2007). 
${ }^{13}$ M. D. Knudson, D. L. Hanson, J. E. Bailey, C. A. Hall, J. R. Asay, and C. Deeney, "Principal Hugoniot, reverberating wave, and mechanical reshock measurements of liquid deuterium to $400 \mathrm{GPa}$ using plate impact techniques," Phys. Rev. B 69(14), 144209 (2004).

${ }^{14}$ H. Kleine, K. Hiraki, H. Maruyama, T. Hayashida, J. Yonai, K. Kitamura, Y. Kondo, and T. G. Etoh, "High-speed time-resolved color schlieren visualization of shock wave phenomena," Shock Waves 14(5-6), 333-341 (2005).

${ }^{15}$ L. M. Barker and R. E. Hollenbach, "Laser interferometer for measuring high velocities of any reflecting surface," J. Appl. Phys. 43(11), 4669-4675 (1972).

${ }^{16}$ J. B. R. Winters, S. N. Bland, S. J. P. Stafford, D. J. Chapman, and D. E. Eakins, "VISAR 'cross-hairs': Simultaneous perpendicular lineimaging VISAR," J. Phys.: Conf. Ser. 500(18), 182044 (2014).

${ }^{17}$ Ya. B. Zel'dovich, Physics of Shock Waves and High-Temperature Hydrodynamic Phenomena (Courier Corporation, 2002).

${ }^{18}$ R. Paul Drake, High Energy Density Physics (Springer-Verlag, Berlin, Heidelberg, 2006).

${ }^{19}$ J. Staudenraus and W. Eisenmenger, "Fibre-optic probe hydrophone for ultrasonic and shock-wave measurements in water," Ultrasonics 31(4), 267273 (1993).
${ }^{20}$ J. E. Parsons, C. A. Cain, and J. B. Fowlkes, "Cost-effective assembly of a basic fiber-optic hydrophone for measurement of high-amplitude therapeutic ultrasound fields," J. Acoust. Soc. Am. 119(3), 1432-1440 (2006).

${ }^{21}$ A. Arvengas, K. Davitt, and F. Caupin, "Fiber optic probe hydrophone for the study of acoustic cavitation in water," Rev. Sci. Instrum. 82(3), 034904 (2011).

${ }^{22}$ J. H. Gladstone and T. P. Dale, "Researches on the refraction, dispersion, and sensitiveness of liquids," Proc. R. Soc. London 12, 448-453 (1862).

${ }^{23} \mathrm{H}$. S. Yadav, "Measurement of refractive index of water under high dynamic pressures,” J. Appl. Phys. 44(5), 2197 (1973).

${ }^{24}$ L. Davison and R. A. Graham, "Shock compression of solids," Phys. Rep. 55(4), 255-379 (1979).

${ }^{25}$ D. T. Blackstock, Fundamentals of Physical Acoustics (John Wiley \& Sons, Inc., 2000).

${ }^{26} \mathrm{~N}$. A. Hawker and Y. Ventikos, "Interaction of a strong shockwave with a gas bubble in a liquid medium: A numerical study," J. Fluid Mech. 701, 59-97 (2012).

${ }^{27}$ M. R. Betney, N. A. Hawker, B. Tully, and Y. Ventikos, "Computational modelling of the interaction of shock waves with multiple gas bubbles in a liquid,” Phys. Fluids 27, 036101 (2015). 